\title{
The Adenosine Hypothesis of Restless Legs Syndrome
}

\author{
Sergi Ferré, $\mathrm{MD}, \mathrm{PhD}^{1,2}$
}

$\mathbf{R}$ ESTLESS LEgs SyndROME (RLS) is a very prevalent neurological disorder. According to the RLS epidemiology, symptoms, and treatment study, $5 \%$ of U.S. and European patients reported experiencing RLS symptoms at least weekly. ${ }^{1}$ RLS can be pathophysiologically separated into two clinical phenomena: deficits of sensorimotor integration that produce akathisia and periodic limb movements during sleep (PLMS) and an enhanced arousal state (hyperarousal). ${ }^{2}$

Akathisia is described as a feeling of restlessness and an urgent need to move. PLMS have very specific characteristics: they consist on repetitive episodes of leg movement activity (at least four in a row) with a duration of up to 10 seconds and an intermovement interval of 5-90 seconds. ${ }^{3}$ Hyperarousal manifests as a short sleep time with episodes of arousals during sleep, related but not caused by PLMS; thus, in about half of all cases, the onset of the episodes of arousal precedes the onset of the leg movements. ${ }^{3}$ Hyperarousal also manifests as a lack of expected profound sleepiness during the day. Thus, the average total sleep time of RLS patients is $<6$ hours and, yet, they do not show significant daytime sleepiness on objective meassures. ${ }^{4}$

Both supraspinal and spinal mechanisms (supraspinal and spinal hyperexcitability) have been invoked to be involved in the pathophysiology of akathisia and PLMS of RLS. ${ }^{5}$ Leg movements with the same qualitative pattern, duration, and periodicity of PLMS have also been reported after acute spinal cord injuries (SCI), in patients with completely absent volitional activity in their legs. ${ }^{6}$ The SCI-induced episodes of leg movements, however, do not a have a circadian dependence and are not associated with episodes of cortical arousal. ${ }^{6}$

Therefore, the spinal component of RLS, spinal hyperexcitability, most probably depends on an altered supraspinal-mediated mechanism, either a hyperfunctioning excitatory or a hypofunctioning inhibitory mechanism. The first situation seems to be the case, since cortical hyperexcitability has been well documented from transcranial magnetic stimulation studies in RLS patients. ${ }^{7}$ Supraspinal mechanisms are also supported by recent findings on RLS genetics indicating that RLS has aspects of a genetically moderated neurodevelopmental disorder involving mainly the corticostriatalthalamic-cortical circuits (see Ref. ${ }^{2}$ for recent review).

Brain iron deficiency (BID) is now well recognized as a main initial pathophysiological mechanism in RLS, which is supported by results obtained from cerebrospinal fluid analysis and from brain imaging and postmortem studies. ${ }^{8}$ BID in rodents during the postweaning period represents a valid pathogenetic model of RLS, since it recapitulates essential biochemical changes observed in RLS, mainly the presynaptic hyperdopaminergic state. This is made evident by the increase in striatal and nigral tyrosine hydroxylase activity and the reduced striatal density of dopamine $\mathrm{D}_{2}$ receptors (D2R), ${ }^{9}$ where downregulation of D2R would represent an adaptive postsynaptic effect of an increased dopamine synthesis and release. ${ }^{8}$

Counterintuitively, in the frame of a presynaptic hyperdopaminergic state, dopamine agonists, such as pramipexole and ropinirole, are clinically effective at reducing the sensorimotor symptoms of RLS. ${ }^{8}$ However, alternative pharmacological agents are being sought, since the main long-term complication of dopamine receptor agonists is the augmentation of RLS symptoms, that is, an overall increase in symptom severity. ${ }^{10}$ After a treatment period of $\sim 10$ years, the prevalence of augmentation is $\sim 50 \%{ }^{10}$

Several clinical findings have also suggested the existence of a presynaptic hyperglutamatergic state in RLS that could be involved not only with the hyperarousal state, but also with the sensorimotor symptoms of RLS. ${ }^{2}$ In fact, glutamatergic mechanisms play a central role in the therapeutic effects of $\alpha_{2} \delta$-ligands, such as gabapentin and pregabalin, which are an alternative treatment to dopamine receptor agonists and are effective for both sensorimotor symptoms and hyperarousal. ${ }^{11}$ Thus, $\alpha_{2} \delta$-ligands target the $\alpha_{2} \delta$-subunits of calcium channels localized in glutamatergic terminals, inhibiting glutamate release. ${ }^{12}$

Recently, we were able to demonstrate the first biochemical correlate of the presynaptic hyperglutamatergic state of RLS in rats with BID: an increased sensitivity of corticostriatal terminals to release glutamate. ${ }^{13}$ Thus,

\footnotetext{
${ }^{1}$ Editor in Chief of the Journal of Caffeine and Adenosine Research.

${ }^{2}$ Integrative Neurobiology Section, National Institute on Drug Abuse, IRP, NIH, DHHS, Baltimore, Maryland.
} 
corticostriatal terminals from BID rats released glutamate with lower frequency of optogenetic stimulation than controls. ${ }^{13}$ Since we had previously demonstrated that corticostriatal glutamate release leads to a local striatal dopamine release, ${ }^{14}$ this could be a mechanism involved in the presynaptic hyperdopaminergic state of RLS. We, therefore, hypothesized that BID-induced hypersensitivity of corticostriatal glutamatergic terminals represents a main pathogenetic mechanism involved in the sensorimotor symptoms of RLS. ${ }^{13}$

Significantly, local application of either the $\alpha_{2} \delta$ ligand gabapentin or the dopamine receptor agonists pramipexole or ropinirole blocked completely glutamate release induced by corticostriatal optogenetic stimulation, both in controls and in animals with BID. ${ }^{13}$ This implied that dopamine receptors and calcium channels localized in corticostriatal terminals might represent key targets for the therapeutic effects of the mainstream drugs prescribed in RLS.

The apparently inexplicable efficacy of dopamine receptor agonists in the frame of a hyperdopaminergic state could then be explained by their action on presynaptic, and not postsynaptic, striatal dopamine receptors. Thus, activation of presynaptic dopamine receptors localized in corticostriatal terminals (more specifically complexes, heteromers, of D2R and dopamine $\mathrm{D}_{4}$ receptors) ${ }^{13}$ should locally inhibit glutamate-dependent dopamine release (without discarding an additional spinal effect; see Ref. ${ }^{15}$ for recent review). An additional implication of our study is that the use of the optogenetic-microdialysis method in BID rats provides an animal model to screen new putative therapeutically effective treatments for RLS. ${ }^{13}$

We recently showed that BID in rodents causes a generalized downregulation of adenosine $A_{1}$ receptors (A1Rs) in the brain. ${ }^{16}$ Based on these results, we have hypothesized that a hypoadenosinergic state secondary to AlR downregulation could be mostly responsible for the hyperglutamatergic and hyperdopaminergic states of RLS that determine the sensorimotor symptoms of RLS as well as the hyperarousal component. ${ }^{2,17}$ In view of the key inhibitory role of A1Rs on striatal glutamate release, A1R downregulation should be mostly responsible for the BID-induced hypersensitivity of corticostriatal glutamatergic terminals. ${ }^{2,17}$

We, therefore, predicted that inhibitors of equilibrative nucleoside transporters, by increasing the striatal extracellular levels of adenosine, could provide a new therapeutic approach for RLS. In fact, encouraging results were obtained with the nonselective ENT1/ENT2 dipyridamole in a recent open trial with RLS patients. ${ }^{18} \mathrm{We}$ were then able to validate the putative role of dipyridamole in our preclinical model and show that it completely blocks optogenetically induced corticostriatal glutamate release in rats with BID, and that the effect of dipyridamole can be counteracted by an A1R antagonist. ${ }^{19}$ Furthermore, we could also demonstrate that A1Rs determine the sensitivity of corticostriatal glutamatergic terminals. Thus, a frequency of optogenetic stimulation that was ineffective at inducing corticostriatal glutamate release in control rats became effective with the local perfusion of a selective A1R antagonist. ${ }^{19}$

Altogether these results support the involvement of the supraspinal striatal A1Rs in the pathogenesis of the sensorimotor symptoms of RLS and the clinical application of inhibitors of adenosine transport in RLS. But A1Rs are also localized in the spinal cord, both in the dorsal horn, controlling the glutamatergic input, ${ }^{20}$ and in the motoneurons of the ventral horn, where they form heteromers with dopamine $D_{1}$ receptors. ${ }^{21}$ These heteromers have been demonstrated to mediate the increased excitability of the spinal motoneuron by the nonselective adenosine receptor antagonist caffeine. ${ }^{21}$

Therefore, if BID is also associated with downregulation of spinal A1Rs, this could be an important mechanism that would contribute to the development of PLMS in RLS. The same mechanism could then explain the supraspinal and spinal hyperexcitability that leads to PLMS in RLS. The term BID should then be extended to central nervous system iron deficiency (CNSID), to also include the spinal cord.

Finally, it is very probable that A1R downregulation is also involved in the hyperarousal of RLS. Adenosine is a main modulator of homeostatic sleep and it mediates the sleepiness induced by prolonged wakefulness. The sleep function of adenosine depends on the ability of adenosine to directly inhibit the function of the multiple interconnected ascending arousal systems, by acting both on their cells of origin and also their broadly targeted cortex, and A1Rs are particularly involved in the adenosine-mediated regulation of these ascending arousal systems. ${ }^{2,22,23}$

Infusion of A1R agonists in the basal forebrain pontomesencephalic tegmentum, lateral hypothalamus, or prefrontal cortex increases sleep, whereas infusion of A1R antagonists in the same areas increases waking (see Ref. ${ }^{23}$ for review). In fact, the A1R has been suggested to be a marker of the homeostatic sleep response, of the need for recovery from lack of sleep (see Ref. ${ }^{17}$ for recent review). Downregulation of A1R has already been shown in the cortex of rodents with $\mathrm{BID},{ }^{16}$ further indicating a generalized phenomenon, which could occur in most areas of the CNS, including the cells of origin of the ascending arousal systems and the spinal cord.

In summary, a significant amount of preclinical and clinical data supports the key role of adenosine in the pathogenesis of RLS, related to a generalized downregulation of A1Rs associated with CNSID. A1R downregulation can constitute the long-sought common pathogenetic link between the apparently nonrelated supraspinal and spinal mechanisms and between the RLS symptomatology secondary to deficits in sensorimotor integration and the hyperarousal.

Several important studies need still to be performed to be able to confirm "The Adenosine Hypothesis of RLS." At the preclinical level, experiments are in progress to demonstrate a CNSID-induced spinal downregulation 
of A1Rs. At the clinical level, A1R downregulation remains to be shown in RLS patients. These experiments can already be performed, since A1R Positron Emission Tomography ligands are already being successfully used in humans. ${ }^{24,25}$ Finally, the clinical efficacy of dipyridamole or other preferably more brain-penetrant and more selective ENT1 inhibitors needs to be fully demonstrated. A larger scale double-blind placebo study with dipyridamole is already in progress.

\section{Acknowledgment}

This study was supported by the intramural funds of the National Institute on Drug Abuse.

\section{Author Disclosure Statement}

No competing financial interests exist.

\section{References}

1. Allen RP, Walters AS, Montplaisir J, et al. Restless legs syndrome prevalence and impact: REST general population study. Arch Intern Med. 2005;165:1286-1292.

2. Ferré S, García-Borreguero D, Allen RP, Earley CJ. New insights into the neurobiology of restless legs syndrome. Neuroscientist. 2019 [Epub ahead of print]; DOI: $10.1177 / 1073858418791763$.

3. Ferri R, Rundo F, Zucconi M, et al. An evidence-based analysis of the association between periodic leg movements during sleep and arousals in restless legs syndrome. Sleep. 2015;38:919-924.

4. Allen RP, Stillman P, Myers AJ. Physician-diagnosed restless legs syndrome in a large sample of primary medical care patients in western Europe: Prevalence and characteristics. Sleep Med. 2010;11:31-37.

5. Trenkwalder C, Paulus W. Restless legs syndrome: Pathophysiology, clinical presentation and management. Nat Rev Neurol. 2010;6:337-346.

6. Salminen AV, Manconi M, Rimpilä V, et al. Disconnection between periodic leg movements and cortical arousals in spinal cord injury. J Clin Sleep Med. 2013;9:1207-1209.

7. Lanza G, Cantone M, Lanuzza B, et al. Distinctive patterns of cortical excitability to transcranial magnetic stimulation in obstructive sleep apnea syndrome, restless legs syndrome, insomnia, and sleep deprivation. Sleep Med Rev. 2015;19:39-50.

8. Earley CJ, Connor J, Garcia-Borreguero D, et al. Altered brain iron homeostasis and dopaminergic function in Restless Legs Syndrome (Willis-Ekbom Disease). Sleep Med. 2014;15:1288-1301.

9. Connor JR, Wang XS, Allen RP, et al. Altered dopaminergic profile in the putamen and substantia nigra in restless leg syndrome. Brain. 2009;132:2403-2412.

10. García-Borreguero D, Cano-Pumarega I. New concepts in the management of restless legs syndrome. BMJ. 2017;356:j104.

11. García-Borreguero D, Patrick J, DuBrava S, et al. Pregabalin versus pramipexole: Effects on sleep disturbance in restless legs syndrome. Sleep. 2014;37:635-643.

12. Dooley DJ, Taylor CP, Donevan S, Feltner D. Ca2+ channel alpha2delta ligands: Novel modulators of neurotransmission. Trends Pharmacol Sci. 2007;28:75-82.
13. Yepes G, Guitart X, Rea W, et al. Targeting hypersensitive corticostriatal terminals in restless legs syndrome. Ann Neurol. 2017;82:951-960.

14. Quiroz C, Orrú M, Rea W, et al. Local control of extracellular dopamine levels in the medial nucleus accumbens by a glutamatergic projection from the infralimbic cortex. J Neurosci. 2016;36:851-859.

15. Trenkwalder C, Allen R, Högl B, et al. Comorbidities, treatment, and pathophysiology in restless legs syndrome. Lancet Neurol. 2018;17:994-1005.

16. Quiroz C, Gulyani S, Ruiqian W, et al. Adenosine receptors as markers of brain iron deficiency: Implications for Restless Legs Syndrome. Neuropharmacology. 2016; 111:160-168.

17. Ferré S, Quiroz C, Guitart X, et al. Pivotal role of adenosine neurotransmission in restless legs syndrome. Front Neurosci. 2018;11:722.

18. Garcia-Borreguero D, Guitart X, Garcia Malo C, CanoPumarega I, Granizo JJ, Ferré S. Treatment of restless legs syndrome/Willis-Ekbom disease with the nonselective ENT1/ENT2 inhibitor dipyridamole: Testing the adenosine hypothesis. Sleep Med. 2018;45:94-97.

19. Ferré S, Quiroz C, Rea W, Guitart X, García-Borreguero D. Adenosine mechanisms and hypersensitive corticostriatal terminals in restless legs syndrome. Rationale for the use of inhibitors of adenosine transport. Adv Pharmacol. 2019 [Epub ahead of print]; DOI:10.1016/ bs.apha.2018.12.005.

20. Li X, Eisenach JC. Adenosine reduces glutamate release in rat spinal synaptosomes. Anesthesiology. 2005;103: 1060-1065.

21. Rivera-Oliver M, Moreno E, Álvarez-Bagnarol Y, et al. Adenosine A(1)-dopamine $\mathrm{D}(1)$ receptor heteromers control the excitability of the spinal motoneuron. Mol Neurobiol. 2018 [Epub ahead of print]; DOI:10.1007/ s12035-018-1120-y.

22. Ferré S. Role of the central ascending neurotransmitter systems in the psychostimulant effects of caffeine. J Alzheimers Dis. 2010;20(Suppl. 1):S35-S49.

23. Brown RE, Basheer R, McKenna JT, Strecker RE, McCarley RW. Control of sleep and wakefulness. Physiol Rev. 2012;92:1087-1187.

24. Mishina M, Ishii K, Kimura Y, et al. Adenosine A(1) receptors measured with (11)C-MPDX PET in early Parkinson's disease. Synapse. 2017;71:e21979.

25. Elmenhorst EM, Elmenhorst D, Benderoth S, Kroll T, Bauer A, Aeschbach D. Cognitive impairments by alcohol and sleep deprivation indicate trait characteristics and a potential role for adenosine A(1) receptors. Proc Natl Acad Sci USA. 2018;115:8009-8014.

Address correspondence to: Sergi Ferré, $M D$, PhD Integrative Neurobiology Section National Institute on Drug Abuse, IRP NIH, DHHS

Triad Building, 333 Cassell Drive Baltimore, MD 21224

E-mail: sferre@intra.nida.nih.gov 